\title{
Strain Analysis in Polycrystalline Diamond under Extreme Conditions
}

Shirin Kaboli ${ }^{1}$, Pamela C. Burnley ${ }^{2}$

1,2. Geoscience Department and High Pressure Science and Engineering Center, University of Nevada Las Vegas, Las Vegas, United States

Diamond has the highest hardness and thermal conductivity of any material, high electrical and wear resistivity as well as excellent thermal and chemical stability. The combination of diamond's unique properties makes it a suitable material for applications in extreme conditions such as high pressure experimentation in a diamond anvil cell (DAC) or a multi-anvil apparatus. Diamonds exist in natural and synthetic forms. Synthetic diamonds are commonly produced by chemical vapor deposition (CVD) techniques. The CVD diamonds typically contain various crystal defects including twins, stacking faults and dislocations, depending on the synthesis environment. These defects control diamond's crystallographic texture and residual stress levels which directly affect the performance of diamond under extreme conditions. Therefore, nanoscale characterization of crystal defects in diamond is important for controlling and producing diamonds with desirable physical and mechanical properties [1].

Transmission electron microscopy (TEM) is used to study crystal defects in diamond thin foils. Thin foils are obtained by cleavage [2], laser cutting [3] or focused ion beam (FIB) [4]. In addition to tedious process of diamond thin foil preparation, all of these techniques inevitably introduce additional defects into the thin foil making it difficult to distinguish them from those that are present in the diamond bulk. To overcome this difficulty, we utilized electron channeling contrast imaging (ECCI) and electron backscatter diffraction (EBSD) on bulk diamond samples at the nanoscale in a field emission gun scanning electron microscope (FE-SEM). There are two main challenges associated with characterization of diamond in a SEM: low channeling contrast in ECCI and low pattern quality in EBSD. In this contribution, we present a novel protocol for surface preparation and microscope operation conditions to perform successful ECCI and EBSD on bulk diamond samples in FE-SEM. Furthermore, we aim to investigate the plasticity of a synthetic polycrystalline diamond used in an insitu synchrotron X-ray deformation experiment conducted at high pressure and high temperature conditions [5].

We present the results of our microstructure characterization using ECCI and EBSD on two diamond samples. The first sample was a synthetic polycrystalline diamond rod of $1 \mathrm{~mm}$ diameter and $500 \mu \mathrm{m}$ thickness with average grain size of $96.2 \pm 33.3 \mu \mathrm{m}$ (Applied Diamond Inc.). Figure 1 shows the asreceived microstructure with grain orientation contrast (a), twins (b), stacking faults (c) and dislocations (d). Our ECCI investigations showed that majority of grains were twinned, indicating that twinning dominated the growth process during the CVD synthesis. The results of EBSD crystal orientation mapping confirmed the presence of $\langle 111\rangle 60^{\circ}$ twins highlighted in red in Figure1f. These results are in agreement with that previously obtained on diamond thin foils by TEM. We then compressed this material in a four step D-DIA experiment [5] conducted in the $\sim 4-7$ GPa pressure range and $~ 200-1100$ ${ }^{\circ} \mathrm{C}$ temperature range. We compared the density and distribution of twins and dislocations in two samples before and after deformation for strain analysis in polycrystalline diamond under these experimental conditions. Our preliminary results show the unprecedented potential of our protocol for surface preparation and microscope operation conditions to perform successful ECCI and EBSD on bulk diamond samples in FE-SEM. We believe that characterization of diamond in SEM can become an 
effective tool for understanding the nature and evolution of crystal defects during synthesis as well as loading of diamond allowing us to understand and control the response of diamond at the nanoscale in various industrial and technological applications.

\section{References:}

[1] T. Liu, PhD Dissertation, Ruhr-University Bochum, (2009).

[2] A. Mussi, D. Eyidi, A. Shiryaev and J. Rabier, Phys. Status Solidi A, 210(2013), p. 191.

[3] J.W. Steeds, A. Gilmore, K.M. Bussmann, J.E. Butler, P.Koidl, Diam. Relat. Mater. 8(1999), p. 996.

[4] F. Tang, Y. Chen and L. Zhang, Appl. Phys. 47(2014), p. 125301.

[5] Y. Wang, B. Durham, I. Getting and D. Weidner, Rev. Sci. Instrum. 74(2003), p. 3002.

[6] This research was sponsored by the National Science Foundation under award NSF-EAR13613399 Additional support was provided to the second author by the National Nuclear Security Administration under the Stewardship Science Academic Alliances program through DOE Cooperative Agreement \#DE-NA0001982. Synchrotron experiments were performed at the Advanced Photon Source which is supported by DOE-BES, under Contract No. DE-AC02-06CH11357 at Sector 6BM which is supported by the Consortium for Materials Properties Research in Earth Sciences under NSF cooperative agreement EAR 06-49658.
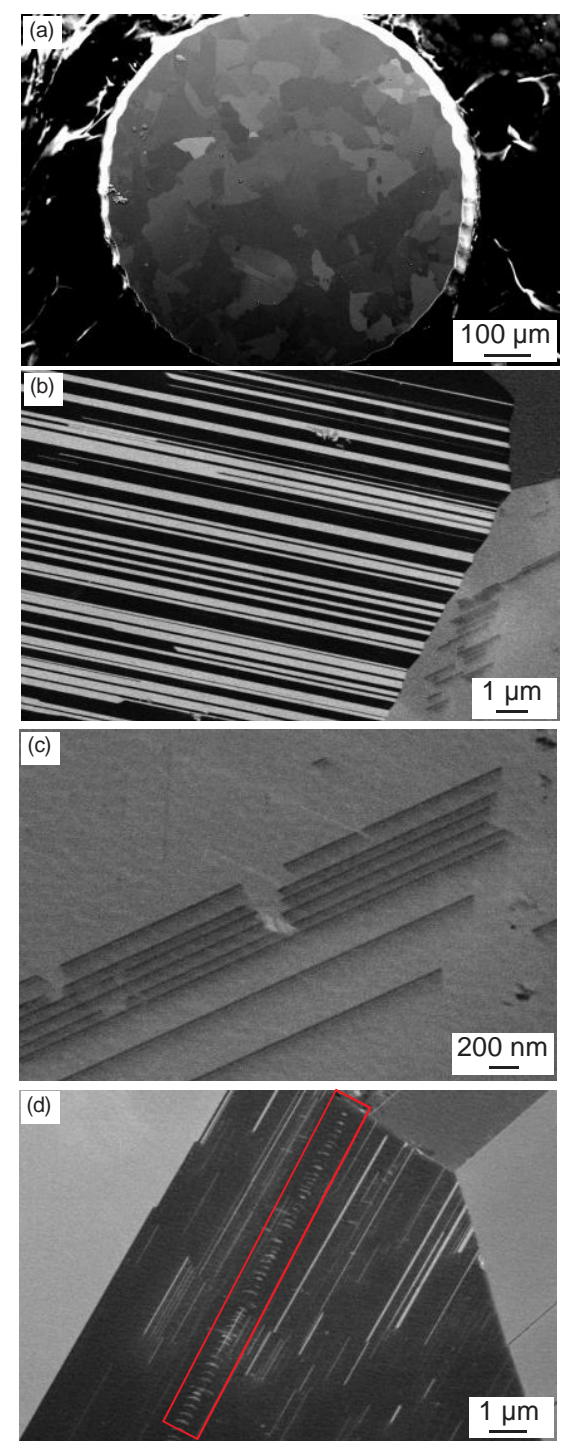
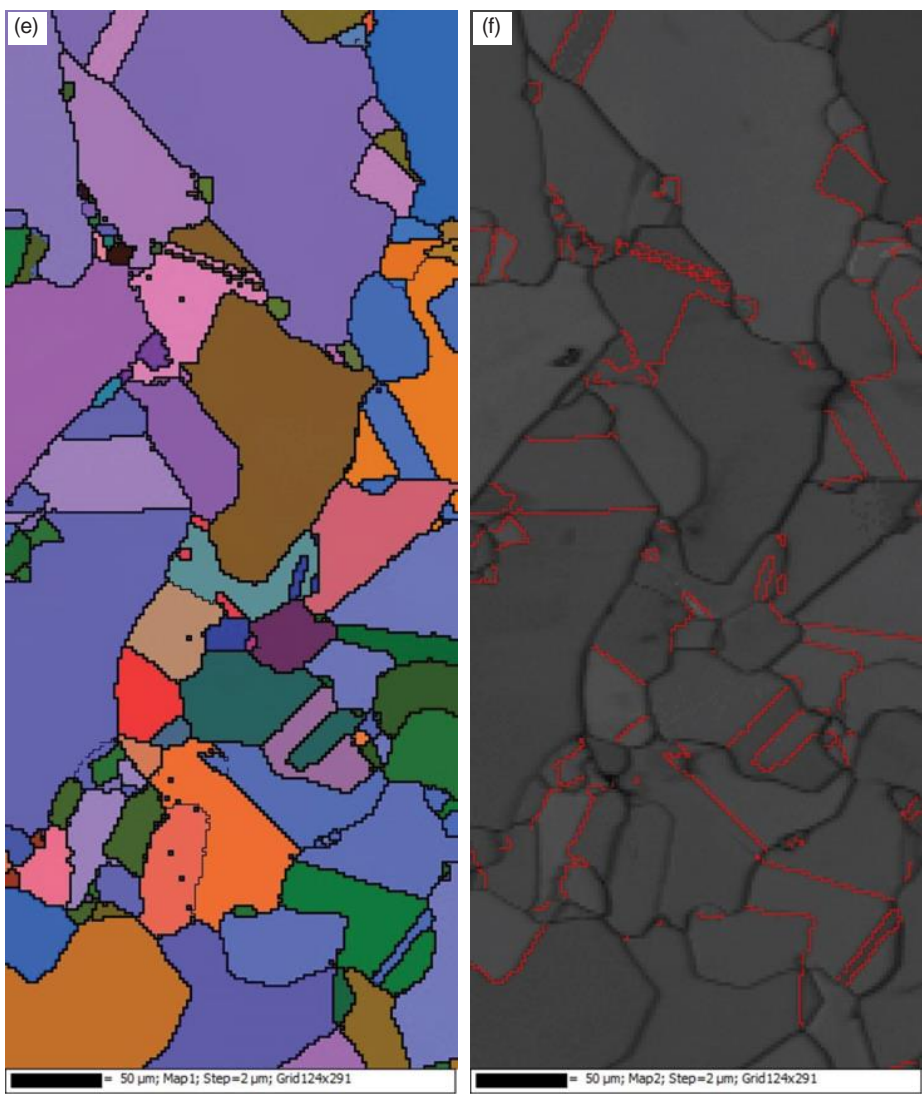

Figure 1. Microstructure of a synthetic polycrystalline diamond before deformation. (a) low magnification grain orientation contrast (b) twins (c) stacking faults, (d) dislocations, (e) all Euler map and (f) band contrast map with highlighted $\langle 111\rangle 60^{\circ}$ twin boundaries displaying the dominant twinning mechanism during the growth of diamond grains. 